\title{
TAMAÑO Y CONFIGURACIÓN SEXUAL DE LOS GRUPOS INFORMALES DE JUEGO EN EL AULA A LO LARGO DE LA EDUCACIÓN PRIMARIA
}

\author{
Victoria Muñoz Tinoco \\ Universidad de Sevilla \\ tinoco@us.es \\ Francisco Juan García Bacete \\ Universitat Jaume \\ fgarcia@uji.es \\ Irene Jiménez Lagares \\ Universidad de Sevilla \\ irene@us.es \\ Ghislaine Marande \\ Universitat Jaume \\ marande@uji.es
}

Recepción Artículo: 27 octubre 2021

Admisión Evaluación: 27 octubre 2021

Informe Evaluador 1: 28 octubre 2021

Informe Evaluador 2: 29 octubre 2021

Aprobación Publicación: 30 octubre 2021

Financiado por: PROYECTO UJI-B2019-29: Evolución, antecedentes y consecuentes del desagrado social expresado por y hacia las chicas. Estudio longitudinal a lo largo de la Educación Primaria

\section{RESUMEN}

El grupo es uno de los contextos de desarrollo más importantes en la infancia. El objetivo de este trabajo es analizar la organización grupal de las niñas a lo largo de la Educación Primaria en términos de centralidad, tamaño y configuración sexual. Se evaluaron 17 aulas de 7 centros educativos de las provincias de Castellón y Sevilla en $1^{0}$ de Educación Primaria ( $N=376,49 \%$ niñas), 2º ( $N=377,50.1 \%$ niñas), $4^{\circ}$ ( $N=375,52 \%$ niñas) y $6^{0}$ ( $N=366$, $51.9 \%)$. Para la identificación de los grupos se utilizó el Social Cognitive Maps (García Bacete y Marande, 2013). El 90\% del alumnado fue identificado por sus iguales como parte de un grupo de juego. La mayor parte de los grupos identificados eran intrasexo, siendo más frecuentes los grupos intrasexo formados por niñas que los formados por niños. En general, los grupos de tamaño mediano son más frecuentes en las chicas y los grandes en los chicos. En $6^{0}$ aumenta el número de grupos por aula, disminuye su tamaño medio y aumenta el porcentaje de díadas y tríadas. Estos resultados muestran la presencia de grupos de juego a lo largo de toda la Educación Primaria, así como la tendencia, mayor en las chicas, a la segregación sexual de los grupos. Las chicas suelen formar parte de grupos más pequeños y su posición es más central, probablemente debido a la preferencia por relaciones más íntimas y exclusivas y por la importancia concedida a la cohesión grupal.

Palabras clave: grupo de iguales; redes sociales; Social Cognitive Maps; diferencias de género 
ABSTRACT

Size and sexual configuration of informal play groups in the classroom throughout elementary education. The peer playgroup is one of the most important developmental contexts in childhood. The aim of this work is to analyze the group organization of girls throughout Primary Education in terms of centrality, size and sexual configuration. Seventeen classrooms from 7 educational centers in the provinces of Castellón and Seville were evaluated in 1st year of Primary Education ( $N=376,49 \%$ girls), $2 \mathrm{nd}(\mathrm{N}=377,50.1 \%$ girls), 4th ( $N$ $=375,52 \%$ girls $)$ and 6 th $(N=366,51.9 \%)$. For the identification of the groups, the Social Cognitive Maps was used (García Bacete and Marande, 2013). Results indicated that $90 \%$ of the students were identified by their peers as part of a play group. Most of the identified groups were intrasex. Female intrasex groups were more frequent than male groups. In general, medium-sized groups are more frequent in for girls and large ones in for boys. In 6th grade, the number of groups per classroom increases, their average size decreases, and the percentage of dyads and triads increases. These results show the presence of playgroups throughout Primary Education, as well as the trend, greater in girls than in boys, towards sexual segregation of the groups. Girls tend to be part of smaller groups and their position is more central, probably due to their preference for more intimate and exclusive relationships and the importance given to group cohesion.

Palabras clave: peer groups; social networks; Social Cognitive Maps; gender differences

\section{INTRODUCCIÓN}

Los expertos en relaciones entre iguales han conceptualizado tres niveles de experiencia social (interacciones, relaciones diádicas y grupos) con implicaciones específicas y diferenciales para el desarrollo (Rubin, Bukowski, Parker y Damon, 1998; Rubin, Bukowski y Laursen, 2009). Cuando los niños y las niñas coinciden en un aula y comienzan a interactuar con sus iguales se establecen relaciones de imitación, reciprocidad o complementariedad que dan lugar a la formación de amistades y grupos.

Este trabajo pretende hacer una primera aproximación a la experiencia de relación grupal. Podemos definir el grupo como una red social en la que las personas interactúan, siendo uno de los contextos de desarrollo fundamentales en la infancia (Xie, Cairns y Cairns, 1999). Formar parte de una red social, así como la posición 0 centralidad que se ocupa en esa red, son experiencias de socialización muy importantes a lo largo de toda la infancia (Gifford-Smith y Brownell, 2003). Sin embargo, el estudio de las redes sociales en edades tempranas ha sido poco abordado por los investigadores. Este desinterés se ha debido, en parte, a la escasez de procedimientos empíricos (Cairns, Gariepy, Kinderman y Leung, 1996).

Existen muchas dimensiones que diferencian a los grupos. Una de las más relevantes, por sus implicaciones para el desarrollo individual, es su estructura (Hitti, Mulvey y Killen, 2011). Sabemos que las relaciones que se establecen entre los iguales y los subgrupos que se forman en el aula no son aleatorios, sino que dependen de factores individuales y contextuales, siendo la proximidad y similitud claves en la configuración de los grupos (Gifford-Smith y Brownell, 2003). En este trabajo vamos a centrarnos en una de las fuentes fundamentales de heterogeneidad grupal: su configuración en términos de tamaño, centralidad y sexo (Rubin, Bukowski y Bowker, 2015).

\section{OBJETIVOS DE LA INVESTIGACIÓN}

Este trabajo pretende realizar un primer acercamiento descriptivo a la formación de los grupos de juego en el centro escolar, analizando la centralidad, el tamaño y la configuración sexual de los grupos de juego en primero, segundo, cuarto y sexto de Educación Primaria.

\section{MUESTRA}

Se evaluaron 17 aulas de 7 centros educativos de las provincias de Castellón y Sevilla a lo largo de los distintos cursos de Educación Primaria. Las relaciones grupales fueron evaluadas en $1^{0}$ de Educación Primaria ( $\mathrm{N}=376,49 \%$ niñas), $2^{\circ}\left(\mathrm{N}=377,50.1 \%\right.$ niñas), $4^{\circ}\left(\mathrm{N}=375,52 \%\right.$ niñas) y $6^{\circ}(\mathrm{N}=366,51.9 \%)$. La edad media en $1^{0}$ de Primaria era de 76.6 meses. 


\section{METODOLOGÍA E INSTRUMENTOS UTILIZADOS}

Para la identificación de los grupos se utilizó el Social Cognitive Maps, un método basado en la percepción directa de niños y niñas respecto a los subgrupos que conforman sus iguales al interactuar en situaciones lúdicas (Cairns, Cairns, Neckerman, Gest y Gariepy, 1988; Cairns et al., 1996; Cairns, Perrin y Cairns, 1985; García Bacete y Marande, 2013). El SCM desarrolla un procedimiento para definir la centralidad de las personas y de los grupos en la red social que es independiente de las preferencias o de las relaciones de amistad, siendo un método completo y preciso para la identificación de las redes sociales (García Bacete y Marande, 2013).

Se pide a los niños que identifiquen "a los compañeros de clase que van juntos muchas veces". A partir de la información proporcionada por todos los miembros del aula, un software específico identifica el número de grupos, la identidad de los individuos que forman parte de cada grupo de iguales y la centralidad en el aula de cada grupo y de cada individuo.

Para este trabajo se utilizaron las siguientes variables:

- Pertenencia de los sujetos a grupo: Aislado, Pertenencia a un Grupo, Multigrupo)

- Configuración sexual del grupo: Intrasexo masculino, intrasexo femenino, mixto

- Tamaño del grupo: díada, tríada, mediano, grande (más de 6 miembros).

- Centralidad del grupo, del alumno en el grupo y en el aula: nuclear, secundaria o periférica.

\section{RESULTADOS ALCANZADOS}

\section{Tamaño y centralidad}

Se identificaron un total de 272 subgrupos de juego, 72 en primero, 62 en segundo, 66 en cuarto y 70 en sexto, con una media de 3.9 grupos por aula y una media de 5.8 miembros por grupo. El $52 \%$ de los grupos son de gran tamaño. El $90 \%$ del alumnado es identificado por sus iguales como parte de un grupo de juego y el $8 \%$ forma parte de dos o más grupos. El $84.5 \%$ de los grupos son nucleares. En cuanto a la centralidad, el $65 \%$ de los sujetos son miembros nucleares de sus grupos y el $58 \%$ son nucleares en el aula.

En cuanto a la evolución con la edad, el número de grupos por aula aumenta ligeramente con la edad, hasta 4.3 grupos por aula en sexto, a la vez que disminuye el tamaño medio de los grupos hasta 5.3 miembros en $6^{0}$ de Primaria. En sexto disminuye el porcentaje de grupos de gran tamaño (6 o más miembros) y aumenta el porcentaje de díadas y tríadas. El porcentaje de alumnado que pertenece a algún grupo se mantiene entre el 85 y el 95\% a las distintas edades, no existiendo diferencias tampoco en el número de grupos a los que se pertenece. La centralidad de los sujetos en sus grupos y en el aula es mayor en $6^{0}$ de Primaria que en cursos anteriores, subiendo hasta el 77 y $70 \%$ respectivamente.

\section{Configuración sexual}

Tres de cada cuatro grupos están formados por niños o niñas del mismo sexo (el $43 \%$ son exclusivamente femeninos, el 33\% masculinos y el 26\% mixtos). En general, los grupos-díadas suelen ser, en su mayoría intrasexo, mientras que en los grupos de mayor tamaño encontramos más presencia de grupos mixtos (en torno al $50 \%)$.

Chicas y chicos forman parte de algún grupo de juego con la misma frecuencia y a todas las edades. En $4^{0}$ de Primaria las chicas son menos exclusivas en su pertenencia a grupos, formando casi el $15 \%$ de ellas parte de dos o más grupos de juego frente al $6.7 \%$ de los chicos. En 60 esta tendencia se invierte y solo el $2 \%$ de las chicas forma parte de más de un grupo, frente al $14 \%$ de los chicos.

Analizando todos los grupos en su conjunto, un porcentaje mayor de chicas tiene una posición central en su grupo (72\%, frente al $64 \%$ de los chicos) y en el aula (64\%) frente al $57 \%$ de los chicos), siendo esta diferencia significativa, aunque con un tamaño de efecto pequeño. Estas diferencias no se mantienen para cada grupo de edad. 


\section{TAMAÑO Y CONFIGURACIÓN SEXUAL DE LOS GRUPOS INFORMALES DE JUEGOEN EL AULA A LO LARGO DE LA EDUCACIÓN PRIMARIA}

A partir de $2^{0}$ las niñas se organizan, preferentemente, en grupos intrasexo de tamaño mediano (en torno al $50 \%$ ), mientras que los grupos intrasexo masculinos son preferentemente de gran tamaño (52\% de los grupos). La presencia de las chicas en los grupos mixtos es menor que la presencia de chicos hasta $4^{0}$ de Primaria, cambiando esta tendencia en $6^{0}$.

\section{DISCUSIÓN}

Estos resultados muestran la estabilidad en la presencia de grupos de juego a lo largo de toda la Educación Primaria. Se observan algunas tendencias con la edad en la configuración de los grupos por tamaño y sexo. Así, aunque todas las posibles configuraciones grupales están presentes en todas las edades, los grupos grandes son más frecuentes en las edades intermedias y las díadas y tríadas tienen más prevalencia a medida que se acerca la adolescencia, probablemente relacionado con la preferencia por relaciones más íntimas y exclusivas a estas edades. En los cursos superiores, las niñas tienden a formar parte de más de un grupo con mayor frecuencia que Ios niños, lo cual apoya la idea de que las niñas tenderían a interactuar secuencialmente en grupos distintos en lugar de interactuar todo el tiempo con los mismos iguales, como suele ocurrir en el caso de los chicos, así como a formar parte de díadas o tríadas conectadas entre sí (Rose y Smith, 2018). La preferencia de chicos por grupos de mayor tamaño apoyaría la hipótesis de Baines y Blacthford (2009), sugiriendo que los niños incorporan con más frecuencia que las niñas a iguales no amigos en sus juegos.

En cuanto a la configuración sexual de los grupos, se confirma la tendencia a la segregación, siendo mayor esta tendencia en las chicas que en los chicos a todas las edades. Esta realidad tiene importantes consecuencias para la socialización de niños y niñas. Los grupos tienden a mantenerse cohesionados a través de la identificación de sus miembros con el mismo y de la oposición a otros grupos. Siendo el sexo una de las características frecuentemente compartidas por el grupo, podemos comprender que los procesos de cohesión grupal fortalezcan la identidad de género y contribuyan a la segregación. Asimismo, sabemos que los procesos de segregación favorecen la aparición de la conducta tipificada. (Rose y Smith, 2006; Ruble, Martin y Berenbaum, 2006).

Un siguiente reto sería analizar las características conductuales y socioemocionales de las chicas y chicos que se implican en grupos mixtos, así como un análisis en profundidad de los integrantes de los grupos para confirmar si la tendencia a la segregación por sexo es mayor que la tendencia a la búsqueda de similitudes.

\section{CONCLUSIONES}

Este estudio confirma una clara tendencia a la segregación sexual en los grupos de juego desde primer curso de Educación Primaria y permite intuir diferencias relevantes en las dinámicas de relación de los grupos formados por niñas y los formados por niños.

Los resultados obtenidos tienen importantes implicaciones para la optimización del desarrollo en la infancia. Desde el modelo de socialización de los iguales parece lógico afirmar que la participación en grupos de juego mixtos podría contribuir a modular los estereotipos y la tipificación sexual. En este sentido, el papel de la escuela y el profesorado al formar los equipos de trabajo es fundamental. Rose y Rudolph (2006) recogen un buen listado de investigaciones que muestran cómo niños y niñas apenas interactúan entre ellos a no ser que el docente u otra figura de autoridad sea quien forme los grupos. Sin embargo, no parece ser suficiente que formar grupos mixtos, pues las interacciones entre niños y niñas son frecuentemente más negativas que las interacciones entre iguales del mismo sexo, estando también reguladas por normas sociales más rígidas (Rodkin, Pearl, Farmer et al.,2003). Estos resultados parecen dar la razón a Maccoby (1998) cuando hablaba de niños y niñas como dos mundos separados. El reto educativo está en facilitar a niños y niñas la exploración de esos "dos mundos", para encontrar en su frontera un espacio confortable.

\section{REFERENCIAS BIBLIOGRÁFICAS}

Baines, E., \& Blatchford, P. (2009). Sex differences in the structure and stability of children's playground social networks and their overlap with friendship relations. British Journal of Developmental Psychology, 27(3), 
743-760. https://doi.org/10.1348/026151008X371114

Cairns, R. B., Cairns, B. D., Neckerman, H. J., Gest, S. y Gariepy, J. L. (1988). Social networks and aggressive behavior: Peer support or peer rejection? Developmental Psychology, 24, 815-823. https://doi.org/10.1037/0012-1649.24.6.815

Cairns, R. B., Gariepy, J. L., Kinderman, T. y Leung, M. C. (1996). Identifying social clusters in natural settings. Unpublished manuscript, University of North Carolina at Chapel Hill.

Cairns, R. B., Perrin, J. E. y Cairns, B. D. (1985). Social structure and social cognition in early adolescence: Affiliative patterns. Journal of Early Adolescence, 5, 339-355.

Garcia Bacete, F. y Marande, G. (2013). Social Cognitive Maps. Un método para identificar los grupos sociales en contextos naturales. Psychosocial Intervention. 22, 61-70. https://doi.org/10.5093/in2013a8.

Gifford-Smith, M. E. y Brownell, C. A. (2003). Childhood peer relationships: social acceptance, friendships, and peer networks. Journal of School Psychology, 41, 235-284. https://doi.org/10.1016/S0022-4405(03)000487

Hitti, A., Mulvey, K. L. y Killen, M. (2011). Social exclusion and culture: The role of group norms, group identity and fairness. Anales de Psicología, 27, 587-599.

Maccoby, E. E. (1998). The two sexes: Growing up apart, coming together. Cambridge: Harvard University Press.

Rodkin, P. C., Pearl, R., Farmer, T. W. y Van Acker, R. (2003). Enemies in the gendered societies of middle childhood: Prevalence, stability, associations with social status, and aggression. New Directions for Child and Adolescent Development, 102, 73-88. https://doi.org/10.1002/cd.90

Rose, A. J. y Rudolph, K. D. (2006). A review of sex differences in peer relationship processes: potential tradeoffs for the emotional and behavioral development of girls and boys. Psychological bulletin, 132(1), 98-131. https://doi.org/10.1037/0033-2909.132.1.98

Rose, A. J. y Smith, R. (2009). Sex Differences in Peer Relationships. En K.H Rubin, W.M. Bukowski y B. Laursen, Handbook of peer interactions, relationships, and groups (pp. 379-393). New York. Guildford Press.

Rose, A. J., \& Smith, R. L. (2018). Gender and peer relationships. In W. M. Bukowski, B. Laursen, \& K. H. Rubin (Eds.), Handbook of peer interactions, relationships, and groups (pp. 571-589). The Guilford Press.

Rubin, K. H., Bukowski, W. M., \& Bowker, J. C. (2015). Children in peer groups. In M. H. Bornstein, T. Leventhal, \& R. M. Lerner (Eds.), Handbook of child psychology and developmental science: Ecological settings and processes (pp. 175-222). John Wiley \& Sons, Inc.

Ruble, D. N., Martin, C. L. y Berenbaum, S. A. (2006). Gender development. En W. Damon, N. Eisenberg (Eds), Handbook of Child Psychology. Vol. 3, Social, emotional, and personality development (6th ed.), (pp. 858932) New York. Wiley.

Xie, H., Cairns, R. B., \& Cairns, B. D. (1999). Social networks and configurations in inner-city schools: Aggression, popularity, and implications for students with EBD. Journal of Emotional and Behavioral Disorders, 7(3), 147-155. https://doi.org/10.1177/106342669900700303. 
